\title{
Mean Field Approach for a Statistical Mechanical Model of Proteins
}

\author{
Pierpaolo Bruscolini \\ Dipartimento di Fisica ES INFM, Politecnico di Torino, \\ C.so Duca degli Abruzzi 24, I-10129 Torino, Italy \\ Fabio Cecconi \\ Dipartimento di Fisica Università "La Sapienza" Ė INFM Unità di Roma1, P.le A. Moro 2, I-00185 Roma, Italy
}

\begin{abstract}
We study the thermodynamical properties of a topology-based model proposed by Galzitskaya and Finkelstein for the description of protein folding. We devise and test three different meanfield approaches for the model, that simplify the treatment without spoiling the description. The validity of the model and its mean-field approximations is checked by applying them to the $\beta$-hairpin fragment of the immunoglobulin-binding protein (GB1) and making a comparison with available experimental data and simulation results. Our results indicate that this model is a rather simple and reasonably good tool for interpreting folding experimental data, provided the parameters of the model are carefully chosen. The mean-field approaches substantially recover all the relevant exact results and represent reliable alternatives to the Monte Carlo simulations.
\end{abstract}

\section{INTRODUCTION}

The free-energy landscape of protein molecules represents the key-information for understanding processes of biomolecular self-organization such as folding, 1,2,3,4 The free-energy landscape, indeed, determines all observable properties of the folding process, ranging from protein stability to folding rates 5.6 .7 .8 .9 Unfortunately, for real proteins, sophisticated all-atom computational methods fail to characterize the free-energy surface, since they are currently limited to explore only few stages of the folding process. As an alternative, one can argue that, taking into account all the complex details of chemical interactions is not necessary to understand how proteins fold into their native state. Rather, elementary models incorporating the fundamental physics of folding, while still leaving the calculation and simulations simple, can reproduce the general features of the free-energy landscape and explain a number of experimental results. This attitude, typical of a statistical mechanics approach, agrees with the widely accepted view that "a surprising simplicity underlies folding" (Baker ${ }^{10}$ ). In fact several experimental ${ }^{11,12,13,14}$ and theoretical studies $15,16,17,18$ indicate the topology of protein native state as a determinant factor of folding. As examples, one can mention the fact that even heavy changes in the sequence that preserve the native state, have a little effect on the folding rates ${ }^{19,20}$ Moreover, the latter are found to correlate to the average contact order, ${ }^{21}$ which is a topological property of native state. Finally, proteins with similar native state but low sequence similarity often have similar transition state ensembles 15.20

Within this context, elementary models $22,23,24,25,26$ which correctly embody the native state topology and interactions, are believed to be useful in describing the energy landscape of real proteins. In this paper, we study one of such topology-based models proposed by Galzitskaya and Finkelstein $(\mathrm{GF}), 27$ which was developed to identify the folding nucleus and the transition state con- figurations of proteins. The model employs a free-energy function with a reasonable formulation of the conformational entropy, which is certainly the most difficult contribution to describe. The energetic term, instead, takes into account only native state attractive interactions. In the original paper, 27 the model was combined with a dynamic programming algorithm to search for transition states of various proteins. To reduce the computational cost of the search, two kinds of approximations were introduced: the protein was regarded as made up of "chain links" of 2-4 residues, that fold/unfold together; besides, only configurations with up to three stretches of contiguous native residues were considered in the search ("triplesequence approximation"). As shown in Ref. 28, the effect of such assumptions is a drastic entropy reduction of the unfolded state and possibly of the transition state. This produces free energy profiles very different from the true ones, thus spoiling the evaluation of $\phi$-values.

Here, we apply the model in a more general statistical mechanical philosophy: namely, we develop three different mean-field approaches of increasing complexity, and compare their prediction with the exact results, obtained by exhaustive enumeration of all the configurations, in the case of a 16-residues-long peptide (C-terminal 41-56 fragment of the streptococcal protein G-B1) ${ }^{29}$ which is known to fold, in isolation, to a $\beta$-hairpin structure. 25 Our main goal here is to test the model against experimental findings and to test the mean-field predictions against the exact results. In the future we will use this knowledge to apply the appropriate mean-field approach to the case of real proteins, for which exhaustive enumeration is unfeasible.

The paper is organized as follows. In the next section, we present and describe the main features of the GF model. In section II, we introduce and discuss three mean-field approximations: the usual scheme, and two other approaches stemming from the knowledge of the exact solution for the Muñoz-Eaton model ${ }^{28}$ In section III, we apply the model and its mean-field approximations to study the folding transition of the $\beta$-hairpin and 
discuss our results.

\section{DESCRIPTION OF GALZITSKAYA-FINKELSTEIN MODEL}

The GF model assumes a simple description of the polypeptide chain, where residues can stay only in an ordered (native) or disordered (non-native) state. Then, each micro-state of a protein with $L$ residues is encoded in a sequence of $L$ binary variables $\mathbf{s}=\left\{s_{1}, s_{2}, \ldots, s_{L}\right\}$, $\left(s_{i}=\{0,1\}\right)$. When $s_{i}=1\left(s_{i}=0\right)$ the $i$-th residue is in its native (non-native) conformation. When all variables take the value 1 the protein is correctly folded, whereas the random coil corresponds to all 0's. Since each residue can be in one of the two states, ordered or disordered, the free energy landscape consists of $2^{L}$ configurations only. This drastic reduction of the number of available configurations represents, of course, a restrictive feature of the model, however, follows the same line of the well known Zimm-Bragg mode ${ }^{30}$ widely employed to describe the helix to coil transition in heteropolymers.

The effective Hamiltonian (indeed, a free-energy function) is

$$
H(\mathbf{s})=\varepsilon \sum_{i<j} \Delta_{i j} s_{i} s_{j}-T S(\mathbf{s}),
$$

where $S(\mathbf{s})$ is given by:

$$
S(\mathbf{s})=R\left[q \sum_{i=1}^{L}\left(1-s_{i}\right)+S_{\text {loop }}(\mathbf{s})\right] .
$$

$R$ is the gas constant and $T$ the absolute temperature. The first term in Eq. (11) is the energy associated to native contact formation. Non native interactions are neglected: this assumption, that can be just tested a posteriori, is expected to hold if, during the folding process, the progress along the reaction coordinate is well depicted on the basis of the native contacts (that is, the reaction coordinate(s) must be related to just the native contacts). Moreover, such progress must be slow with respect to all other motions, so that all non-native interaction can be "averaged-out" when considering the folding process ${ }^{9}$ $\Delta_{i j}$ denotes the element $i, j$ of the contact matrix, whose entries are the number of heavy-atom contacts between residues $i$ and $j$ in the native state. Here we consider two amino-acids in contact, when there are at least two heavy atoms (one from aminoacids $i$ and one from $j$ ) separated by a distance less than $5 \AA$. The matrix $\Delta$ embodies the geometrical properties of the protein. Notice that, in the spirit of considering the geometry more relevant than the sequence details, every (heavy) atom-atom contact is treated on equal footing: the chemical nature of the atoms is ignored, together with a correct account for the different kind of interactions.

The second term in (1) is the conformational entropy associated to the presence of unfolded regions along the chain, and vanishes in the native state.
More precisely the first term in Eq. (2) is a sort of "internal" entropy of the residues, that can be attributed to the ordering of the main and side-chains' degrees of freedom upon moving from the coil to the native state. Indeed, $q R$ represents the entropic difference between the coil and the native state of a single residue, as can be noticed by considering that in the fully unfolded state the first and last term vanish, and the entropy is given by $q L R$.

The quantity $R S_{\text {loop }}$ in Eq. (2), instead, is the entropy pertaining to the disordered closed loops protruding from the globular native state ${ }^{31}$ it reads:

$$
S_{\text {loop }}(\mathbf{s})=\sum_{i<j} J\left(r_{i j}\right) s_{i} s_{j} \prod_{k=i+1}^{j-1}\left(1-s_{k}\right) .
$$

According to Ref. 27, we take $\mathrm{s}^{32}$

$$
J\left(r_{i j}\right)=-\frac{5}{2} \ln |i-j|-\frac{3}{4} \frac{r_{i j}^{2}-a^{2}}{A a|i-j|} .
$$

In this context a disordered loop is described by a strand of all "0" s between two "1" s: for instance the configuration 11000000111100011 contains two loops involving 6 and 3 residues respectively. The product in expression (3) warrants that only uninterrupted sequences of "0" can contribute to the loop entropy. The configuration of a disordered loop going from residues $(i+1)$ to $(j-1)$, with $i$ and $j$ in their native positions, is assimilated to a gaussian chain of beads ( $C_{\alpha}$ atoms) with end-to-end distance $r_{i j}$, the latter being the distance between $\mathrm{C}_{\alpha}$ atoms of residues $i$ and $j$ in the native state. The parameters $a=3.8 \AA$ and $A=20 \AA$ are the average distance of consecutive $C_{\alpha}$ 's along the chain and persistence length respectively. Other forms for $S_{\text {loop }}$ could also be used (see, e.g. Ref. 22); yet, here we are interested in evaluating the original GF model and devising good mean-field approximations to it, and we will not discuss this subject any further. The interested reader may refer to the original articles ${ }^{27.31}$ for a derivation of Eq. (4).

\section{MEAN FIELD APPROACHES TO THE GF MODEL}

Mean field approach (MFA) is certainly the first attempt to investigate the thermodynamical properties of complex systems, because it provides a qualitative picture of the phase diagram that in many cases is only partially modified by more accurate refinement of the theory. In its variational formulation, MFA, for a system with Hamiltonian $H$ and corresponding free-energy $F$, starts from the Bogoliubov-Feynman inequality

$$
F \leq F_{0}+\left\langle H-H_{0}\right\rangle_{0},
$$

where $H_{0}$ is a solvable trial Hamiltonian $F_{0}$ is the corresponding free-energy, both depending on free parameters 
$\mathbf{x}=\left\{x_{1} \cdots x_{L}\right\}$ (variational parameters). Such parameters have to be chosen to minimize the second member of (5) to get the minimal upper bound of $F$ and accordingly its better approximation. This method defines a variational free-energy

$$
F_{\text {var }}=F_{0}+\left\langle H-H_{0}\right\rangle_{0},
$$

whose minimization leads to the self consistent equations that in their general form read

$$
\left\langle\frac{\partial H_{0}}{\partial x_{l}}\right\rangle_{0}\left\langle H-H_{0}\right\rangle_{0}-\left\langle\left(H-H_{0}\right) \frac{\partial H_{0}}{\partial x_{l}}\right\rangle_{0}=0,
$$

with $l=1, \ldots, L$. We implement different versions of the MFA for the GF model that differ each from the other by the choice of the trial Hamiltonian.

\section{A. Standard Mean Field Approach (MFA1)}

To implement the standard MFA for the GF model, we regard the free energy function (II) as an effective Hamiltonian.

The trial Hamiltonian we choose, corresponds to applying an inhomogeneous external field with strengths $\mathbf{x}=\left\{x_{1}, \ldots, x_{L}\right\}$ along the chain

$$
H_{0}=\sum_{i=1}^{L} x_{i} s_{i},
$$

with $x_{i}$ to be determined by minimizing the variational free-energy ${ }^{33}$

$$
F_{\text {var }}(\mathbf{x}, T)=\sum_{i=1}^{L} f_{0}\left(x_{i}, T\right)+\left\langle H-H_{0}\right\rangle_{0},
$$

where $\sum_{i} f_{0}\left(x_{i}, T\right)$ is the free energy associated to $H_{0}$,

$$
f_{0}\left(x_{i}, T\right)=-\frac{1}{\beta} \ln \left\{1+\exp \left(-\beta x_{i}\right)\right\} .
$$

Thermal averages, performed through the Hamiltonian $H_{0}$, factorize $\left\langle s_{i} s_{j} \ldots s_{k}\right\rangle_{0}=\left\langle s_{i}\right\rangle_{0}\left\langle s_{j}\right\rangle_{0} \ldots\left\langle s_{k}\right\rangle_{0}$. The approximate average site "magnetization" $m_{i}=\left\langle s_{i}\right\rangle_{0}$ depends only on the field $x_{i}$, and is given by

$$
m_{i}=\frac{\partial F_{0}}{\partial x_{i}}=\frac{1}{1+\exp \left(\beta x_{i}\right)} .
$$

Instead of working with external fields $x_{i}$ 's, it is more intuitive to use the corresponding "magnetizations" $m_{i}$ 's, writing $F_{v a r}$ as a function of the $m_{i}$ 's. Due to the choice of $H_{0}$, Eq. (8), and to the expression (111), evaluating the thermal average $\langle H\rangle_{0}$ amounts to replacing, in the Hamiltonian Eq. (11), each variable $s_{i}$ by its thermal average $m_{i}$ (11). In the end we get:

$$
F_{v a r}(\mathbf{m}, T)=\varepsilon \sum_{i j} \Delta_{i j} m_{i} m_{j}-T S(\mathbf{m})+R T \sum_{i=1}^{L} g\left(m_{i}\right),
$$

where $g(u)=u \ln (u)+(1-u) \ln (1-u)$ and $S(\mathbf{m})$ is obtained from Eq. (2) by substituting $s_{i} \rightarrow m_{i}$. The last term corresponds to $F_{0}-\left\langle H_{0}\right\rangle_{0}$ in Eq. (6): it is the entropy associated to the system with Hamiltonian $H_{0}$ and is the typical term that stems from this kind of MFA 33 Carrying out the minimization of function (12) with respect to $\mathbf{m}$ leads to self-consistent equations:

$$
g^{\prime}\left(m_{i}\right)=\varepsilon \sum_{j} \Delta_{i j} m_{j}-R T\left(q-\frac{\partial S_{l o o p}(\mathbf{m})}{\partial m_{i}}\right) .
$$

Equations (13) can be solved numerically by iteration and provide the optimal values of the magnetizations that we denote by $\mathbf{m}^{*}$. Once the set of solutions $\mathbf{m}^{*}$ is available, we can compute the variational free-energy $F_{v a r}\left(\mathbf{m}^{*}\right)$ that represents the better estimation of the system free-energy $F$.

In a mean-field approach, the (connected) correlation function between residues $i$ and $j$,

$$
c_{i j}(T)=\left\langle s_{i} s_{j}\right\rangle-\left\langle s_{i}\right\rangle\left\langle s_{j}\right\rangle,
$$

can be recovered through a differentiation of $F_{\text {var }}(\mathbf{m}, T)$ :

$$
c_{i j}^{-1}(T)=\beta\left(\frac{\partial F_{v a r}}{\partial m_{i} \partial m_{j}}\right)_{\mathbf{m}^{*}},
$$

where the subscript indicates that the derivative is evaluated on the solutions $\mathbf{m}^{*}$. Explicitating each term of $F_{v a r}$ we obtain the expression

$$
c_{i j}^{-1}(T)=\frac{\delta_{i j}}{m_{i}^{*}\left(1-m_{i}^{*}\right)}+\varepsilon \beta \Delta_{i j}-\left(\frac{\partial^{2} S_{l o o p}(\mathbf{m})}{\partial m_{i} \partial m_{j}}\right)_{\mathbf{m}^{*}} .
$$

The correlation function matrix is given by the inversion of above matrix.

\section{B. Second Mean Field Approach (MFA2)}

The quality of the MFA improves when we make a less naive choice for $H_{0}$. One of the possible $H_{0}$ is suggested by the Muñoz-Eaton model25.34.35 that was proven to be fully solvable in Ref. 28. In fact, even if the two models are not equivalent, there is an interesting formal relationship between that model and the present one. In the Muñoz-Eaton model, the (effective) energy of a configuration results from the contributions coming from the stretches of contiguous native residues it presents, plus an entropic contribution from each of the non-native residues ${ }^{28.34}$

Here the effective energy Eq. (1) boils down to the contributions of stretches of contiguous non-native residues (the loops), plus the sum of pairwise interactions of native residues. This latter term makes the model harder to solve than Muñoz-Eaton's one. If we neglect this interaction, and replace it with a residue-dependent contribution, the model can be mapped on the Muñoz-Eaton 
model. Indeed, a trial Hamiltonian of the kind:

$$
H_{0}(\mathbf{x})=\sum_{i=1}^{L} x_{i} s_{i}-T S(\mathbf{s}),
$$

with $S\left(\left\{s_{i}\right\}\right)$ given by Eqs. (23), can be recast as $H_{0}=$ $C+H_{M E}$ upon the substitution $s_{i} \rightarrow\left(1-s_{i}\right)$, where $C=\sum x_{i}$ is a constant, and

$$
H_{M E}=\sum_{i<j}\left(u_{i j} \prod_{k=i}^{j} s_{k}\right)+\sum_{i} \mu_{i} s_{i}
$$

with

$$
\begin{aligned}
u_{i j} & =-R T\left[J_{i-1, j+1}-J_{i, j+1}-J_{i-1, j}+\left(1-\delta_{i, j-1}\right) J_{i, j}\right], \\
\mu_{i} & =-R T\left(q+J_{i-1, i+1}\right)-x_{i},
\end{aligned}
$$

(here $J_{i, j}=J\left(r_{i j}\right)$ of Eq. (4); $J_{0, i}=J_{i, L+1}=0$ ). Now the trial Hamiltonians reads formally as the MuñozEaton Hamiltonian: see Eq. (1) of Ref. 28, where the symbol $m_{i}$ was used instead of $s_{i}$.

Hence, we choose Eq. (17) as the trial Hamiltonian, and write down the mean field equations Eq. (7):

$\tilde{\varepsilon_{l}}\left[\sum_{i<j} \varepsilon_{i, j} \Delta_{i, j}\left(C_{i, j, l}-C_{l} C_{i, j}\right)-\sum_{i=1}^{L} x_{i}\left(C_{i, l}-C_{i} C_{l}\right)\right]=0$

for $l=1, \ldots, L$. These equations involve the functions

$$
\begin{gathered}
C_{i}=\left\langle s_{i}\right\rangle_{0} \\
C_{i, j}=\left\langle s_{i} s_{j}\right\rangle_{0} \\
C_{i, j, l}=\left\langle s_{i} s_{j} s_{l}\right\rangle_{0}
\end{gathered}
$$

where averages are evaluated by the same transfer matrix technique as in Ref. 28.

Using the fact that CVM is exact for the Muñoz-Eaton Model, it can also be proven that the three-point functions $C_{i, j, l}$ can be written as a function of the two-point ones: $C_{i, j, l}=C_{i, j} C_{j, l} / C_{j}$, for $i<j<l^{36}$. This greatly reduces the computational cost of minimizing the variational free energy and makes the approach particularly suitable for long polypeptide chains.

Correlations $c_{i j}$ could still be evaluated as in Eq. (15), but now the dependence of $F_{v a r}$ upon $m_{i}$ cannot be worked out explicitly, and the derivatives must be evaluated resorting to the dependence on the fields $x_{j}$ : namely $\partial F_{\text {var }} / \partial m_{i}=\sum_{j}\left(\partial x_{j} / \partial m_{i}\right)\left(\partial F_{\text {var }} / \partial x_{j}\right)$. However, this entails to evaluate the four-point averages $\left\langle s_{i} s_{j} s_{k} s_{l}\right\rangle_{0}$, with a consequent relevant computational cost, for this reason, we will not pursue this strategy in the following.

\section{Third Mean Field Approach (MFA3)}

In the previous MFA version, the entropic term was treated exactly while the energy contribution was very roughly approximated. This new version aims to better incorporate the energy contributions and we shall see that results are in excellent agreement with the exact solution obtained by exact enumeration on the $\beta$-hairpin. We consider the set of configurations of the proteins with $M$ native residues $(M=0, \ldots, L)$. We then take as the trial Hamiltonian

$$
H_{0}(\mathbf{x})=\sum_{M=0}^{L} \delta\left(M-\Sigma_{i} s_{i}\right) H_{0}^{(M)}(\mathbf{x}),
$$

where $\delta(\bullet)$ is the Kronecker delta, and $H_{0}^{(M)}$ is the Hamiltonian restricted to the configurations with $M$ natives:

$$
H_{0}^{(M)}(\mathbf{x})=\sum_{i=1}^{L} \tilde{\varepsilon}_{i} x_{i} \frac{M-1}{L-1} s_{i}-T S(\mathbf{s})
$$

with $\tilde{\varepsilon_{i}}=(1 / 2) \sum_{j=1}^{N} \varepsilon_{i, j} \Delta_{i, j}$. Each residue $i$, in a generic configuration with $M$ native residues, feels an interaction $\tilde{\varepsilon_{i}}$ which it would feel in the native state, weakened by a factor $(M-1) /(L-1)$ (accounting for the fact that not all the residues are native), times the external field $x_{i}$, to be fixed by the mean field procedure.

This scheme is useful for taking correlations into account in a better way than in the usual MFA, so to gain some insight on the parts of the chain that fold first and to investigate folding pathways. In this framework the partition function is:

$$
\mathcal{Z}_{0}=\sum_{M=0}^{L} Z^{(M)}=\sum_{M=0}^{L} \sum_{\left\{s_{i}=0,1\right\}}^{(M)} \exp \left(-\beta H_{0}^{(M)}\right),
$$

where the symbol $(M)$ above the sum indicates that the sum is restricted to configurations with $M$ native residues. The mean field equations (77) reads

$\tilde{\varepsilon_{l}}\left[\sum_{i<j} \varepsilon_{i, j} \Delta_{i, j}\left(\mathcal{C}_{i, j, l}^{\prime}-\mathcal{C}_{i, j} \mathcal{C}_{l}^{\prime}\right)-\sum_{i=1}^{L} x_{i} \tilde{\varepsilon}_{i}\left(\mathcal{C}_{i, l}^{\prime \prime}-\mathcal{C}_{i}^{\prime} \mathcal{C}_{l}^{\prime}\right)\right]=0$, 
for each $l$, where

$$
\begin{gathered}
\mathcal{C}_{\bullet}=\sum_{M=1}^{L} C_{\bullet}^{(M)} \\
\mathcal{C}_{\bullet}^{\prime}=\sum_{M=1}^{L} \frac{(M-1)}{(L-1)} C_{\bullet}^{(M)} \\
\mathcal{C}_{\bullet}^{\prime \prime}=\sum_{M=1}^{L} \frac{(M-1)^{2}}{(L-1)^{2}} C_{\bullet}^{(M)} \\
C_{i}^{(M)}=\frac{1}{\mathcal{Z}_{0}} \sum_{\left\{s_{i}\right\}}^{(M)} s_{i} \exp \left(-\beta H_{0}^{(M)}\right) \\
C_{i, j}^{(M)}=\frac{1}{\mathcal{Z}_{0}} \sum_{\left\{s_{i}\right\}}^{(M)} s_{i} s_{j} \exp \left(-\beta H_{0}^{(M)}\right) \\
C_{i, j, l}^{(M)}=\frac{1}{\mathcal{Z}_{0}} \sum_{\left\{s_{i}\right\}}^{(M)} s_{i} s_{j} s_{l} \exp \left(-\beta H_{0}^{(M)}\right)
\end{gathered}
$$

are the contributions to the correlation associated to configurations with $M$ native residues. The transfermatrix method applied in Ref. 28 allows keeping track separately of the contributions coming from the configurations with a given total number of native residues, therefore it is possible to evaluate exactly the partition functions $\mathcal{Z}_{0}^{(M)}$, and all the averages Eq. (27) involved in the mean field equations Eq. (26). The computational cost is relevant, though: in fact, due to the necessity of evaluating all $C_{i, j}^{(M)}$ and some $C_{i, j, l}^{(M)}$ (the ones actually occurring in Eq. (26) $), O\left(L^{6}\right)$ elementary multiplications are required. As far as correlations $c_{i j}$ are concerned, the same discussion of the MFA2 case holds.

\section{THE $\beta$-HAIRPIN}

We compare the MFA results with numerical simulations on the $\beta$-hairpin, the fragment $41-56$ of the naturally occurring protein GB1 (2GB1 in the Protein Data Bank) 29 This peptide has been widely studied experimentally, $25,37,38$ through all-atom simulations $39.40,41$ and simplified models $\frac{25.34,42}{\text { Thus it }}$ represents a good test for the validity of the model and its approximations. Since the $\beta$-hairpin contains only $L=16$ aminoacids, we can carry out exact enumeration over the $2^{16}=65536$ possible configurations to compute explicitly the partition function

$$
Z(\beta)=\sum_{\left\{s_{i}\right\}} \exp (-\beta H)
$$

of the model. Once the function $Z$ is known, all the thermal properties are available and it is possible to completely characterize the thermal folding of the hairpin peptide. However, first, we have to adjust the model free

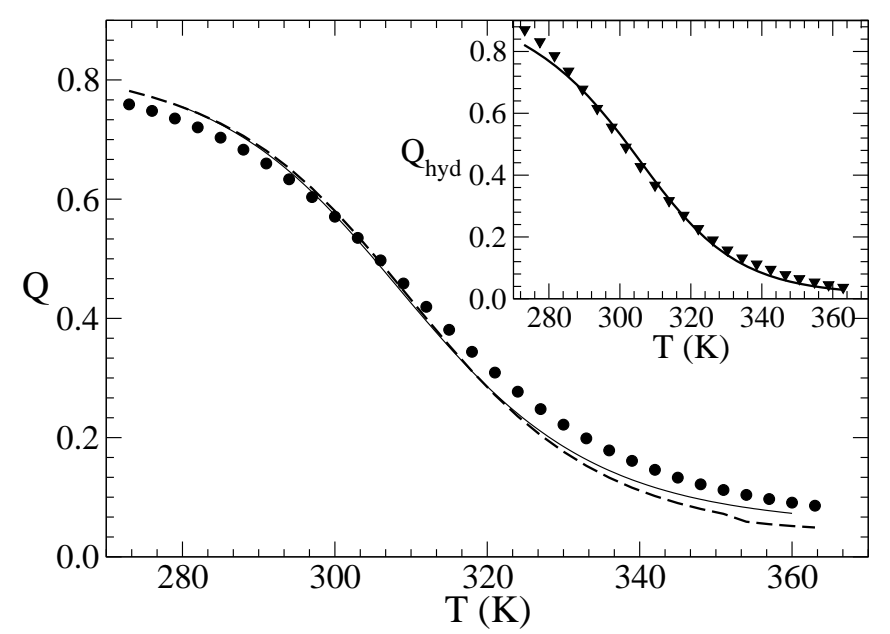

FIG. 1: Fraction of native residues $Q$ (see 30 ) during thermal folding, according to the GF model. Full dots are the exact result obtained by exhaustive enumeration. Dashes and full lines indicate MFA1 and MFA3 approximations, respectively. Inset: Fit of the hydrophobic cluster $(W 43-Y 45-F 52-$ $V 54$ ) population $Q_{h y d}$ (solid) to the experimental data from ${ }^{25}$ (triangles).

parameters $\varepsilon$ and $q$ to reproduce experimental data on the hairpin equilibrium folding. Experimental results on tryptophan fluorescence, ${ }^{34}$ show that, in the folded state, the $99 \%$ of molecules contain a well formed hydrophobic cluster made of Trp43, Tyr45, Phe52 and Val54. In the model, the formation of the hydrophobic cluster is described by the behaviour of the four-points correlation function $Q_{h y d}=\left\langle s_{3} s_{5} s_{12} s_{14}\right\rangle$ (notice that, here and in the following, residues are renumbered from 1 to 16 , instead of 41-56). The choice of the model parameters $q=2.32$ and $\varepsilon=-0.0632(\mathrm{kcal} / \mathrm{mol})$ provides the best fit of $Q_{h y d}$ to the behavior of the experimental fraction of folded molecules (cfr. inset of Fig. 1 with Fig. 3 of Ref. 34). We can now assess the goodness of the model and its mean-field approximations, by comparing their predictions with the experimental results and simulations.

Averages and correlations within the mean-field schemes will be evaluated as follows: for MFA1, the selfconsistent mean-field equations (13) are solved by iteration, substituting an arbitrary initial value for $\mathbf{m}$ at the right-hand side of Eq. (13), evaluating $m_{i}$ from the lefthand side, and substituting again the latter value in the right-hand side, until convergence is achieved.

In the present case, this procedure converges quickly to two different solutions (depending on the starting values of the fields), corresponding to different phases: the folded one $\left(m_{i} \sim 1\right)$ at low temperature and the unfolded $\left(m_{i} \sim 0\right)$ at high temperature. Starting from the unfolded phase and lowering the temperature the solution of Eqs. (13) remains trapped into a set of misfolded metastable states. Only at temperatures well below the folding temperature $T_{F}$ the solution collapses into the 


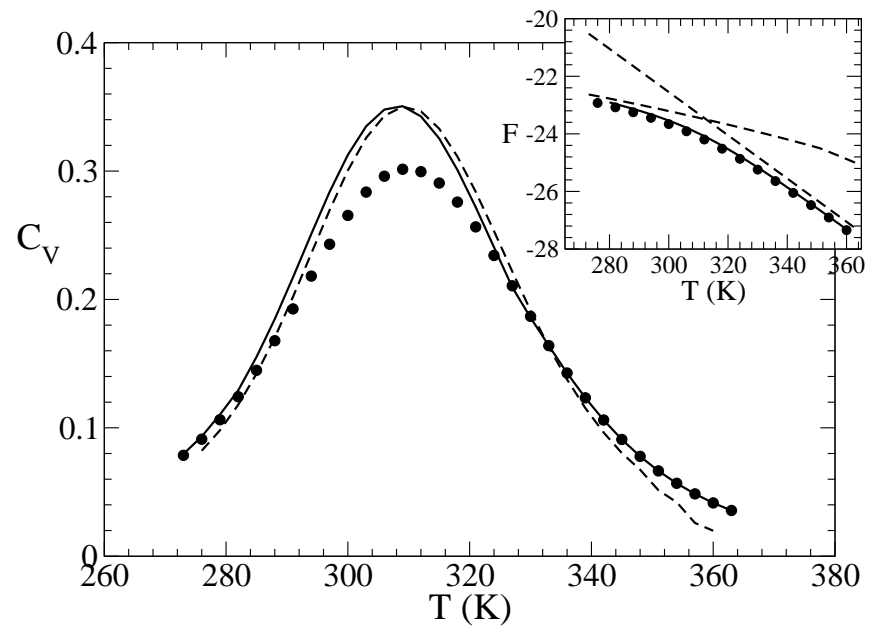

FIG. 2: Comparison between MFA and the exact enumeration. Behaviour of specific heat $\left(\mathrm{kcal} \mathrm{mol}^{-1} \mathrm{~K}^{-1}\right)$ and freeenergy ( $\mathrm{kcal} \mathrm{mol}^{-1}$ ) with temperature, as obtained by the exact enumeration of the GF model applied to the hairpin. Dots indicate the exact results, while dashed and solid lines correspond to MFA1 and MFA3, respectively. In the inset, the mean-field free energies and the exact free energy are plotted against temperature rendering conventions as before. Notice the crossing of two branches of MFA1 at the transition temperature.

one representing the folded state. The opposite happens when the temperature is increased starting from the folded phase. This is a typical scenario of first-order like transitions, which is reproduced by the mean field approach. The situation is well illustrated by the behaviour of the mean field free-energy, which exhibits two branches $F_{1}(T)$ and $F_{2}(T)$ as shown by the dashed lines in the inset of Fig. 2] The intersection of the two branches defines the mean-field folding temperature. At a given temperature, the free-energy of the protein is obtained by selecting the minimum of the two branches

$$
F(T)=\min \left\{F_{1}(T), F_{2}(T)\right\} .
$$

In this approximation other observables present a jump at transition: this reflects the fact that in the thermodynamic limit (here corresponding to infinitely long proteins), only the solution with the lowest free-energy would be physical. To take into account finite-size effect, we decide to introduce an interpolating formula to deal with a continuous quantity:

$$
\langle O\rangle=\frac{\mathrm{e}^{-\beta F_{1}}\langle O\rangle_{1}+\mathrm{e}^{-\beta F_{2}}\langle O\rangle_{2}}{\mathrm{e}^{-\beta F_{1}}+\mathrm{e}^{-\beta F_{2}}},
$$

where $\langle O\rangle_{1}$ and $\langle O\rangle_{2}$ are the averages of the observable in the above mentioned branches. In this way we compute the average magnetization (i.e. the fraction of correctly folded residues) of the protein:

$$
Q=\frac{1}{L} \sum_{i=1}^{L}\left\langle s_{i}\right\rangle
$$

as well as its energy $\langle E\rangle$. In the latter case $\langle E\rangle_{1},\langle E\rangle_{2}$ are evaluated as $\langle E\rangle_{\alpha}=\partial\left(\beta F_{\alpha}\right) / \partial \beta$.

Differentiating the energy with respect to the temperature, we get the specific heat, reported in Fig. 2] Notice that this is the correct recipe to take into account also the contributions to the specific heat coming from the change of the native fraction of molecules: the alternative one, obtained with the direct application of Eq. (29) to the specific heats $C_{v}^{1}=\partial\langle E\rangle_{1} / \partial T$ and $C_{v}^{2}=\partial\langle E\rangle_{2} / \partial T$, would neglect the change in the number of folded molecules, and account only for the variations of the energy within the pure native or unfolded state. For the same reason, Eq. (29) is not useful to match the correlation functions $c_{i j}$ evaluated on the two branches. It would yield only a linear superposition of the $c_{i j}$ 's relative to native and unfolded states, while the correct functions should account for the contributions coming from all the configuration space.

Coming to MFA2, we observe that it keeps exactly into account the entropic term Eq. (2). Yet, solving the mean-field equations yields again two different solutions at each temperature. Thus, MFA2 presents the same kind of problems in characterizing the folding transition states as MFA1. This is why in the following we will present results just for MFA1 and MFA3, that behave in a substantially different way.

With MFA3, in fact, a unique set of fields $\mathbf{x}(T)$ is observed, independent of the starting values, for any temperature in the interesting range around the transition, and no empirical connection rule Eq. (29) is required. Moreover, at odds with MFA1 and MFA2, the difference between $F_{v a r}$ and $F_{0}$ in Eq. (6) happens to be negligible at all the relevant temperatures: $F_{0}$ is a very good approximation to $F_{v a r}$. This suggests that the correct correlation functions, which would be very hard to evaluate, can be replaced by the ones involving averages with the trial Hamiltonian $H_{0}: c_{i j} \simeq\left\langle s_{i} s_{j}\right\rangle_{0}-\left\langle s_{i}\right\rangle_{0}\left\langle s_{j}\right\rangle_{0}$. Thus, within MFA3 it is possible to give a substantially correct characterization both of the native and unfolded states, and of the folding nucleus.

In Fig. 1 we plot $Q$ of Eq. (30) as a function of the temperature, for the original model, for MFA1 (with the help of Eq. (29) ) and MFA3. At low temperatures, where the protein assumes its native state, $Q=1$, while $Q \sim 0$ in coil configurations (i.e. at high temperatures). Mean field approximations appear to be slightly more "cooperative" than the original model, according to their steeper sigmoidal shape. The temperature at which $Q=1 / 2$ is an estimate of the folding temperature: we have $T_{F} \sim 306-306.5 \mathrm{~K}$ for the original model, and $T_{F} \sim 305 \mathrm{~K}$ for both MFA1 and MFA3.

In Fig. 2 we plot the specific heat:

$$
C_{v}=\frac{\left\langle E^{2}\right\rangle-\langle E\rangle^{2}}{R T^{2}}=\frac{\partial U}{\partial T}
$$

and the free energy. The peak of $C_{v}$, which provides another definition for the folding temperature, occurs around $T_{F} \sim 309.5 \mathrm{~K}$ for the exact model and its mean 


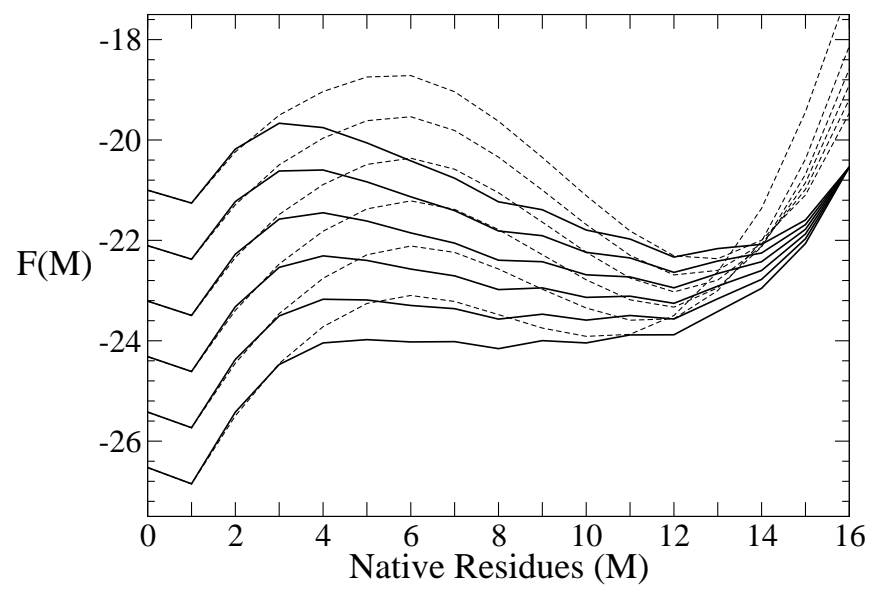

FIG. 3: Free energy landscape for the hairpin, i.e. plot the free-energy $\left(\mathrm{kcal} \mathrm{mol}^{-1}\right.$ ) of the system vs. the number of native residues $M$. Solid lines: exact results for the GF model; dotted lines: MFA3. Temperatures are $285 \mathrm{~K}, 300 \mathrm{~K}, 315 \mathrm{~K}$, $330 \mathrm{~K}, 345 \mathrm{~K}, 360 \mathrm{~K}$, from top to bottom.

field approximations. Notice that MFA1 and MFA3 substantially recover the position of the exact peak, even if the transition appear a little sharper in the mean-field cases.

The above estimates of the folding temperatures are somewhat higher than the experimental ones, $T_{F} \sim 298$ $\mathrm{K} 25$ and $T_{F} \sim 295.3 \mathrm{~K} \stackrel{37}{ }$. Interestingly, $T_{F}$ appears to be higher than the experimental value also for "united atom" simulation 41 ( $T=308 \mathrm{~K}$ in the Go-model case, $T=333 \mathrm{~K}$ with the full potential introduced in that paper), and for all-atoms simulations ${ }^{39}$

Free-energy profiles, for various temperatures, are plotted in Fig. 3 versus the number of native residues $M$, that we use as the folding reaction coordinate. Profiles suggest that the $\beta$-hairpin folding is well described by a two state process, i.e. $F(M)$ exhibits two minima separated by a barrier that has to be overcome in order to reach the native/unfolded state. Notice, though, that this doesn't rule out the possibility that folding might not be a two-state process in this case: this could happen if the number of native residues $M$ was not a good reaction coordinate ${ }^{43}$ Other alternative order parameters should be considered, in addition to $M$, to completely ascertain the nature of the transition.

The comparison between exact and mean-field results reveals that the barrier appears to be overestimated in the MF scheme, where it is also shifted towards higher values of the reaction coordinate: again, the MFA appears to be more cooperative than the original model. Notice however that the free-energy and position of the native and unfolded minima, and hence the stability gap, are correctly recovered, especially at temperatures close to transition (i.e. the second and third plots from top down).

Another interesting characterization of the folding pathway comes from the temperature behavior of the

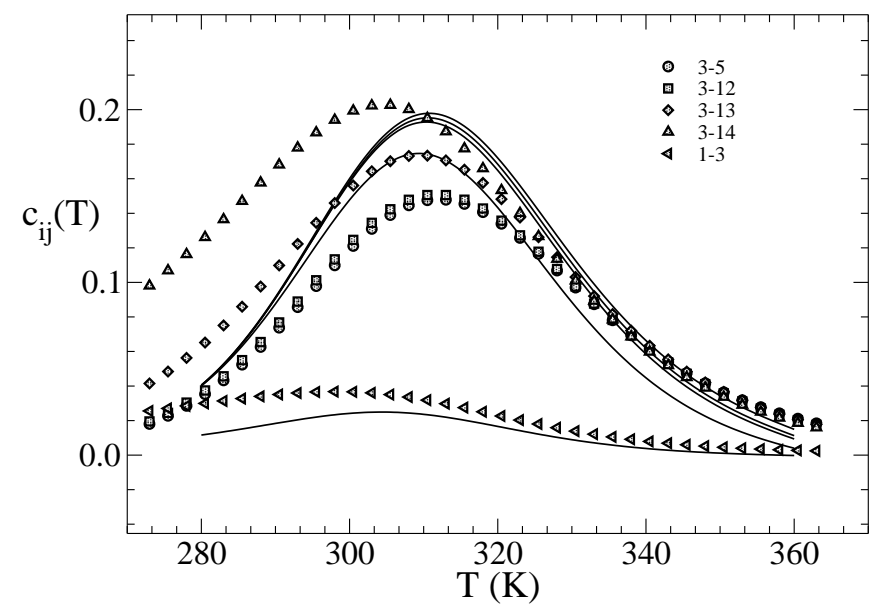

FIG. 4: Temperature behavior of the correlation function $c_{i j}(T)$ of native contacts involving the Tryptophan (Trp45). Symbols correspond to the exact solution, while solid lines indicate the MFA3 results for the contacts $1-3,3-5,3-12$, 3-13, 3-14, from bottom to top.

pairwise correlation functions between residues

$$
c_{i j}(T)=\left\langle s_{i} s_{j}\right\rangle-\left\langle s_{i}\right\rangle\left\langle s_{j}\right\rangle,
$$

that provides an insight on the probability of contact formation during the thermal folding, as shown in Refs. 4445

In fact, each function $c_{i j}(T)$ develops a peak at a characteristic temperature, which can be regarded as the temperature of formation/disruption of the contact $i-j$. In Fig. 4 we plot the correlation functions between Trp45 and residues to which it is in native interaction. The height of each peak indicates the relevance of the contact from a thermodynamical point of view ${ }^{44.45 .46 .47}$ Thus, each contact turns out to be characterized thermodynamically by the location (temperature) and the height of the corresponding peak. This provides a criterion for ranking contacts in order of temperature and relevance (see Refs. 44 45). For example, at the folding temperature $T_{F}$, the contacts that mainly contribute to the folding transition must be searched among those with the characteristic temperature located around $T_{F}$ and with highest peak of $c_{i j}$. Correlation analysis for the hairpin is summarized in Table【 where we report the temperature and the height of correlation function peaks, between residues which share a native contact. Contacts are sorted in temperature and whenever a tie occurs the sorting runs over the heights of the peaks. In this way, we can have a picture of how contacts are established during the thermal folding. Assuming that the order of contact stabilization upon decreasing the temperature reflects the order of formation during folding, this is also a qualitative indication of the folding pathway.

We see, from the first three columns of Table \ that GF model predicts that the $\beta$-hairpin folding begins with the formation of contacts $6-11$ and $6-9,9-11$ and $6-8$, located in the region between the turn $(8-9)$ and the 


\begin{tabular}{|rll||rll|}
\hline \hline \multicolumn{3}{c}{ Exact } & \multicolumn{3}{c|}{ Mean Field } \\
Contact & $\mathrm{T}_{\text {char }}$ & Corr. Peak & Contact & $\mathrm{T}_{\text {char }}$ & Corr. Peak \\
\hline \hline $6-11$ & 316.5 & 0.21457 & $6-11$ & 312.0 & 0.21582 \\
$6-9$ & 316.5 & 0.21346 & $9-11$ & 312.0 & 0.21553 \\
$9-11$ & 316.5 & 0.21243 & $6-9$ & 312.0 & 0.21441 \\
$6-8$ & 316.5 & 0.18262 & $6-12$ & 311.5 & 0.21535 \\
$5-11$ & 315.5 & 0.20457 & $4-6$ & 311.5 & 0.21449 \\
$6-12$ & 315.5 & 0.20214 & $5-11$ & 311.5 & 0.21449 \\
$7-9$ & 315.5 & 0.18462 & $11-13$ & 311.5 & 0.21447 \\
$6-10$ & 315.5 & 0.16488 & $5-12$ & 311.5 & 0.21414 \\
$5-12$ & 315.0 & 0.21670 & $4-12$ & 311.5 & 0.21031 \\
$5-7$ & 315.0 & 0.18461 & $5-13$ & 311.5 & 0.21007 \\
$10-12$ & 315.0 & 0.15697 & $5-7$ & 311.5 & 0.19342 \\
$7-10$ & 314.5 & 0.15048 & $7-9$ & 311.5 & 0.19198 \\
$8-10$ & 314.5 & 0.14339 & $6-8$ & 311.5 & 0.18361 \\
$11-13$ & 314.0 & 0.17713 & $10-12$ & 311.5 & 0.17117 \\
$4-6$ & 314.0 & 0.17575 & $6-10$ & 311.5 & 0.16936 \\
$4-12$ & 313.5 & 0.19244 & $4-13$ & 311.0 & 0.20685 \\
$5-13$ & 313.5 & 0.18681 & $7-10$ & 311.0 & 0.15091 \\
$3-5$ & 312.0 & 0.14805 & $8-10$ & 311.0 & 0.14385 \\
$4-13$ & 310.5 & 0.20932 & $3-5$ & 310.5 & 0.19785 \\
$3-12$ & 310.5 & 0.15090 & $3-12$ & 310.5 & 0.19526 \\
$12-14$ & 310.5 & 0.14416 & $3-13$ & 310.5 & 0.19284 \\
$3-13$ & 309.5 & 0.17364 & $12-14$ & 310.5 & 0.19111 \\
$4-14$ & 309.0 & 0.16487 & $4-14$ & 310.0 & 0.18529 \\
$2-4$ & 307.0 & 0.08024 & $3-14$ & 309.5 & 0.17464 \\
$2-13$ & 306.5 & 0.08037 & $1-15$ & 308.5 & 0.08026 \\
$13-15$ & 306.0 & 0.05241 & $2-4$ & 307.5 & 0.08349 \\
$3-14$ & 304.5 & 0.20284 & $2-13$ & 307.0 & 0.08081 \\
$2-14$ & 300.0 & 0.11045 & $2-14$ & 306.0 & 0.07482 \\
$1-3$ & 298.5 & 0.03678 & $13-15$ & 306.0 & 0.04143 \\
$1-14$ & 296.5 & 0.03570 & $2-15$ & 305.0 & 0.01189 \\
$14-16$ & 290.5 & 0.09773 & $1-3$ & 304.5 & 0.02493 \\
$2-15$ & 273.0 & 0.12746 & $1-14$ & 304.5 & 0.01934 \\
$1-15$ & 273.0 & 0.05005 & $14-16$ & 291.0 & 0.00293 \\
\hline \hline & & & & & \\
\hline \hline
\end{tabular}

TABLE I: Ranking of native contacts according to characteristic temperature and height of the correlation peak. ${ }^{45}$ Contacts 1-16 and 2-16 have been neglected: they yield bad results because they are not stable even in the experimental native structure ${ }^{34}$ The first three columns refer to the exact solutions, the others to MFA3 results.

hydrophobic cluster. Then, upon lowering the temperature, the folding proceeds with the formation of the other contacts that complete $\beta$-hairpin structure. This is at odds with the results of more detailed models and simulations ${ }^{39.40 .41}$ predicting that folding starts with the formation of contacts between the side chains of the hydrophobic cluster, and proceeds with the stabilization of the hydrogen bonds in the loop region (there is no agreement on the order of hydrogen-bonds formation, though). GF model predictions are different also from those of the Muñoz-Eaton model, ${ }^{28.34}$ where the hairpin starts folding from the loop region and proceeds outwards in a zipper fashion. Experimental results relying on point mutations 38 witness the importance of the hydrophobic residues 3, 5, 12 and, to a minor extent, 14, in stabilizing the hairpin structure. Remarkably, contacts between residues $6,9,11$ appear to be partially present also in denaturing conditions $\underline{38}$

It is interesting to notice, however, that, according to Table 【 contacts $3-13,4-14,3-12,12-14,4-13$ of the hydrophobic cluster are mainly established around the folding temperature, which suggests that also in GF model the hydrophobic cluster plays a central role. This is a nice feature of the model because it is consistent with the experimental evidence (fluorence signal) for the formation of the tryptophan hydrophobic environment at the folding.

The estimation of correlation functions provided by MFA3 is only in qualitative agreement with exact results (see Fig. 4): contacts are formed in a narrower range of temperatures, and a direct comparison would be meaningless. However we can ask what kind of information can be extracted from the mean-field results, wondering, for instance, whether the ranking of contact formation provided by MFA3 is "statistically equivalent" to that given by exact solution. Thus, we apply the Spearman rank-order correlation test $\underline{48}$ This test amounts to computing Spearman correlation

$$
R_{s}=1-\frac{6 \sum_{i=1}^{n}\left(x_{i}-y_{i}\right)^{2}}{n\left(n^{2}-1\right)}
$$

where $x_{i}$, and $y_{i}$ are the integer indicating the positions of the $i$-th contact in the two ranking respectively. The parameter, $R_{s}$ is 1 when the order in the two ranks is the same $x_{i}=y_{i}$, while $R_{s}=-1$, when the order is reverse $x_{i}+y_{i}=n$. For data in Table \ we obtain the value $R_{s}=0.902$, that has a probability $P<10^{-6}$ to take place if the null hypothesis of uncorrelated ranks holds. This indicates that the order between the contacts obtained with exact and approximate methods is extremely significative: the mean-field approach basically recovers the correct order of contact formation and relevance as obtained with the true original model.

One of the most important experimental techniques for characterizing the folding nucleus of a protein (more precisely of a protein with two-state folding) consists in the evaluation of $\Phi$-values. $\Phi$-values measure the effect of "perturbation" introduced in a protein by site-directed mutagenesis $\underline{49}$ A mutation performed on the $i$-th residue may affect the thermodynamics and kinetics, by altering the free-energy difference between the native and unfolded state (i.e. the stability gap) or the height of the folding/unfolding barrier. Its effect is quantified through the $\Phi$-value, defined as

$$
\Phi_{i}(T)=\frac{\Delta\left(\Delta F_{\ddagger U}\right)}{\Delta\left(\Delta F_{N U}\right)},
$$




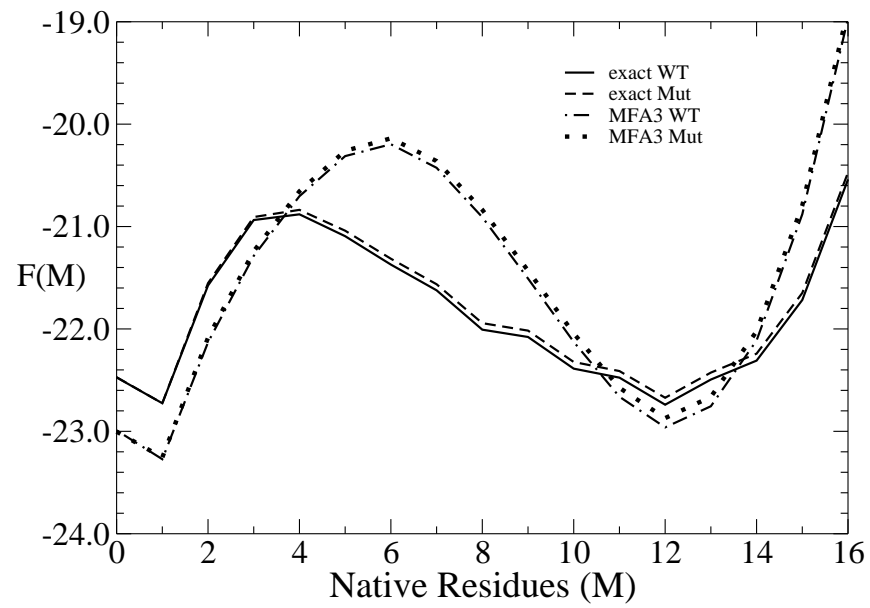

FIG. 5: Variation on the free-energy profile induced by the perturbation on all the interactions involving the sixth residue (Asp46) of the Hairpin. The variation (in kcal mol${ }^{-1}$ ) is computed for both the exact solution and MFA3, at the respective temperatures of equal populations of the native and unfolded basins. Solid and dashed lines indicate wild-type and mutated profiles respectively for the exact solution; dot-dashed and points refer to wild-type and mutated profile respectively in the MFA3.

where $\Delta F_{\ddagger U}=F_{\ddagger}-F_{U}, \Delta F_{N U}=F_{N}-F_{U}$, and $\Delta\left(\Delta F_{\ddagger U}\right)$ and $\Delta\left(\Delta F_{N U}\right)$ are the variations, with respect to the wild type protein, introduced by the mutation in the folding barrier and stability gap. Experimentally, $\Delta\left(\Delta F_{\ddagger U}\right)$ is derived from the changes in the kinetic rates induced by different denaturant concentrations, while $\Delta\left(\Delta F_{N U}\right)$ is extracted from the changes in the equilibrium population. $\Phi$-values are different for different mutations of a residue; in any case, a $\Phi$-value close to one implies that the mutated residue has a native-like environment in the transition state and hence is involved in the folding nucleus. A value close to zero, instead, indicates that the transition state remains unaffected by the mutation, and hence the mutated residue is still unfolded at transition.

In our theoretical description, a mutation at site $i$ is simulated by weakening the strength of the couplings $\varepsilon \Delta_{i j}$ of $1 \%$ between residue $i$ and the others. We choose a small perturbation because we cannot predict what kind of rearrangements in the local structure, and hence in the contact map, a true residue-to-residue mutation would involve. Our choice warrants that the effect of mutation remains local and does not disrupt completely the state. In figure [5. we show the effect of a "mutation" of the sixth residue (Asp46) on the free-energy profiles.

To evaluate the $\Phi$-values, we compute the variations in free energy profiles induced by each mutation, for the exact solution and MFA3. $F_{U}$ and $F_{N}$ are evaluated as $F_{U, N}=-R T \ln Z_{U, N}$, where $Z_{U}, Z_{N}$ are, respectively, the partition functions restricted to unfolded and native basins in the free-energy profile, i.e. the regions to the left and right of the top of the barrier in Fig. 3 $F_{\ddagger}$ is the free-energy of the top of the barrier. Through expres-

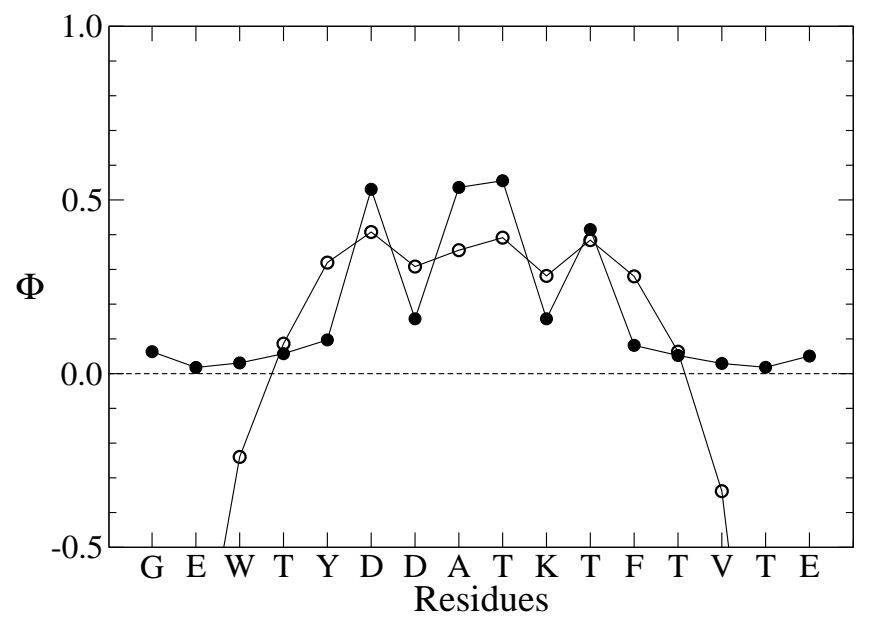

FIG. 6: Effects of "mutations" as measured by $\Phi$-values on each residue. Full circles: $\Phi$-values from the exact solution; open circles: $\Phi$-values within MFA3 approach. The temperatures are in both cases those whereby $F_{U}=F_{N}$. Results depend only slightly on temperature, anyway.

sion Eq. (34) we obtain the $\Phi$-values for each residue. In Fig. [6 we report the $\Phi$-value distributions. There is a good overall correlation between the profiles, that increase and decrease together. This is a further confirmation that the relevant features of the model are conserved when applying the MF approach. Mean-field results yield smoother profiles, as it could be expected. The ends of the hairpin are characterized by low $\Phi$-values, that become negative for MFA3: this would correspond to mutations that increase the stability gap but decrease the barrier, or vice-versa. According to these results, the folding nucleus would be made up by residues $6,8,9,11$, which is in contrast with the already mentioned simulations.

\section{CONCLUSIONS}

In this work we developed and discussed three different mean-field schemes for the Galzitskaya-Finkelstein model, that represent valid ways to deal with the model for characterizing the thermodynamical properties of a protein and its folding pathway as well. These approaches offer viable alternatives both to the procedure proposed by Galzitskaya and Finkelstein, ${ }^{27}$ and to MC simulations, that become computationally demanding for long polymers and usually affected from the sampling problems. We applied the model to the $\beta$-hairpin fragment 41-56 of the GB 1 protein, since, for this simple system, mean field results can be compared with a brute force solution of the model, and both can be checked against experimental data and simulation published by other groups.

Our results suggest that, as far as specific heat and simple thermodynamic quantities are concerned, the stan- 
dard mean-field MFA1 is enough to yield correct results, provided that one uses the recipe Eq. (29) to connect the two branches of the solution. For more sophisticated quantities like free-energy profiles, correlations and $\Phi$ values, MFA3 is to be preferred, since it correctly recovers the main features of the exact solution. The hope is that mean-field results are still representative of the exact ones in the case of longer and more complex proteins, where the latter cannot be evaluated.

GF model itself yields results that are somewhat in contrast with the MC and MD simulations on more detailed models for the hairpin. This discrepancy is probably due to the extreme simplicity of the hamiltonian Eq. (11), where no distinction is made among the different kinds of interactions, such as hydrogen-bonds, side chain hydrophobicity, and so on. Indeed, we expected that a model accounting just for the topology of the native state will not score very well when applied to the $\beta$-hairpin, where detailed sequence information is relevant 38 Predictions of the model could possibly improve if these elements were taken into account.

\section{Acknowledgments}

We thank A. Maritan, C. Micheletti, A. Flammini and A. Pelizzola for their suggestions and useful discussions about the model. F.C. thanks A. Vulpiani and U.M.B. Marconi and acknowledges the financial support of Cofin Murst 2001 on "Fisica Statistica di Sistemi Classici e Quantistici". P.B. acknowledges the financial support of Cofin Murst 2001 "Approccio MeccanicoStatistico ai Biopolimeri".
1 J.D. Bryngelson and P.G. Wolynes, Proc. Natl. Acad. Sci. USA 84, 7524 (1999).

2 P.G. Wolynes, J.N. Onuchic and D. Thirumalai, Science 267, 1619 (1995).

3 J.N. Onuchic, Z. Luthey-Schulten and P.G. Wolynes, Ann. Rev. Phys. Chem. 48, 545 (1997).

${ }_{5}^{4}$ K.A. Dill and H.S. Chan, Nature Struct. Biol. 4, 10 (1997).

${ }^{5}$ H.S. Chan and K.A. Dill, Proteins: Struct. Funct. and Genetics 30, 2 (1998).

${ }^{6}$ V. Muñoz and L. Serrano, Fold. Des. 1, R71 (1996).

7 S.E. Jackson, Fold. Des. 3, R81 (1998).

8 A.R. Fersht, Proc. Natl. Acad. Sci. USA 92, 10869 (1995).

9 M. Karplus, J. Phys. Chem. 104, 11 (2000).

10 D. Baker, Nature, 40539 (2000).

11 D.S. Riddle, V.P. Grantcharova, J.V. Santiago, E. Alm, I. Ruczinski and D. Baker Nature Struct. Biol. 6, 1016 (1999).

12 A. Fersht, Proc. Natl. Acad. Sci. USA 97, 1525 (1999).

13 J.C. Martinez and L. Serrano, Nature Struct. Biol. 6, 1010 (1999).

14 E.J. Miller, K.F. Fischer and S. Marqusee, Proc. Natl. Acad. Sci. USA 99, 10359 (2002).

15 K.W. Plaxco, K.T. Simons, I. Ruczinski and D. Baker, Biochemistry 39, 11177 (2000).

16 C. Micheletti, J.R. Banavar, A. Maritan, and F. Seno, Phys. Rev. Lett. 82, 3372 (1999).

17 C. Clementi, H. Nymeyer and J.N. Onuchic, J. Mol. Biol. 298, 973 (2000).

18 C. Clementi, P.A. Jennings and J.N. Onuchic, Proc. Natl. Acad. Sci. USA, 97, 5871 (2000).

19 D.S. Riddle, J.V. Santiago, S.T. Bray-Hall, N. Doshi, V.P. Grantcharova, Q. Yi and D. Baker, $\mathrm{Na}$ ture Struct. Biol. 4, 805 (1997).

20 F. Chiti, N. Taddei, P.M. White, M. Bucciantini, F. Magherini, M. Stefani and C.M. Dobson, $\mathrm{Na}$ ture Struct. Biol. 6, 1005 (1999).

21 K.W. Plaxco, K.T. Simons and D. Baker, J. Mol. Biol. 277, 985 (1998).

22 E. Alm and D. Baker, Proc. Natl. Acad. Sci. USA 96, 11305 (1999).

23 N. Go, Macromolecules 9, 535 (1976).
24 N. Go and H. Abe, Biopolymers 20, 991 (1981).

25 V. Munoz, P.A. Thompson, J. Hofrichter and W.A. Eaton, Nature 390, 196 (1997).

26 C. Micheletti, J.R. Banavar, and A. Maritan, Phys. Rev. Lett. 87, 088102 (2001).

27 O. V. Galzitskaya and A. V. Finkelstein, Proc. Natl. Acad. Sci. USA 96, 11299 (1999).

28 P. Bruscolini and A. Pelizzola, Phys. Rev. Lett. 88, 258101 (2002).

29 The sequence is GEWTYDDATKTFTVTE.

30 B.H. Zimm and J.K. Bragg, J. Chem. Phys. 31, 526 (1959).

31 A.V. Finkelstein and A.Ya. Badretdinov, Mol. Biol. 31, 391 (1997).

32 There is a misprint in Eq. (2) of the original paper ${ }^{27}$ : $d$ should read $a$ (O. Galzitskaya, private communication).

33 M. Plischke and B. Bergersen, Equilibrium Statistical Physics, World Scientific, Singapore 1989.

34 V. Munoz, E.R. Henry, J. Hofrichter and W. A. Eaton, Proc. Natl. Acad. Sci. USA 95, 5872 (1998).

35 V. Munoz and W.A. Eaton, Proc. Natl. Acad. Sci. USA 96, 11311 (1999).

36 We gratefully acknowledge Dr. A. Pelizzola for this observation.

37 S. Honda, N. Kobayashi, and E. Munekata, J. Mol. Biol. 295, 269 (2000).

38 N. Kobayashi, S. Honda, H. Yoshii, and E. Munekata, Biochemistry 39, 6564 (2000).

39 A.R. Dinner, T. Lazaridis, and M. Karplus, Proc. Natl. Acad. Sci. USA 96, 9068 (1999).

40 V.S. Pande and D.S. Rokhsar, Proc. Natl. Acad. Sci. USA 96, 9602 (1999).

41 D.K. Klimov and D. Thirumalai, Proc. Natl. Acad. Sci. USA 97, 2544 (2000).

42 C. Guo, H. Levine and D.A. Kessler, Phys. Rev. Lett. 84, 3490, (1999).

43 Indeed, this is what Karplus and coworkers suggest in Ref. 39; yet other results $\frac{40,41}{}$ do not support this view.

44 F. Cecconi, C. Micheletti, P. Carloni, and A. Maritan, Proteins: Struct. Funct. and Genetics 43, 365 (2001).

45 C. Micheletti, F. Cecconi, A. Flammini and A. Maritan Protein Sci. 11, 1878 (2002). 
46 A. Scala, N.V. Dokholyan, S.V. Buldyrev and H.E. Stanley, Phys. Rev. E, 63, 032901 (2001).

47 G. Settanni, T.X. Hoang, C. Micheletti and A. Maritan Biophys. J. 83, 3533 (2002).

48 W.H. Press, B.P. Flannery, S.A. Teukolsky, and W.T. Vet- terling, Numerical Recipes, (Cambridge University Press: 1993).

49 A. Fersht, Enzyme and Structure Mechanism, 2nd edition, W.H Freeman and Company, New York 1985. 